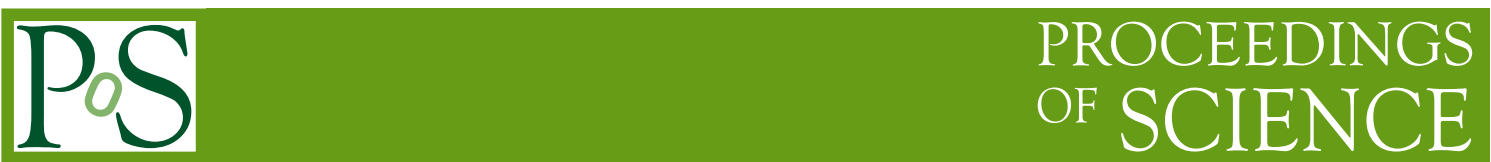

\title{
CBM/FAIR capabilities for charm and dilepton studies
}

Peter Senger ${ }^{* \dagger}$

GSI Helmholtzzentrum für Schwerionenforschung $\mathrm{GmbH}$

E-mail: p.senger@gsi.de

The Compressed Baryonic Matter (CBM) experiment will be one of the major scientific activities at the future Facility for Antiproton and Ion Research (FAIR) in Darmstadt. The goal of the CBM research program is to explore the QCD phase diagram in the region of high baryon densities using high-energy nucleus-nucleus collisions. This includes the study of the equation-of-state of nuclear matter at high densities, and the search for the deconfinement and chiral phase transitions. The CBM detector is designed to measure both bulk observables with large acceptance and rare diagnostic probes such as charmed particles and vector mesons decaying into lepton pairs. The layout of the CBM detector and its physics performance for charm and dilepton measurements will be discussed.

5th International Workshop on Critical Point and Onset of Deconfinement - CPOD 2009,

June 08 - 122009

Brookhaven National Laboratory, Long Island, New York, USA

${ }^{*}$ Speaker.

${ }^{\dagger}$ Supported by EU FP7 Hadronphysics2 


\section{Introduction}

The investigation of nuclear matter at extreme conditions, i.e. at high temperatures and/or at high baryon densities, is one of the most challenging fields of modern physics. At very high temperatures but low net baryon densities, i.e. where the number of particles and antiparticles are almost equal, Lattice-QCD calculations find a crossover transition from hadronic to partonic matter, leading to predictions for the critical temperature which vary between 150 to $190 \mathrm{MeV}[1,2,3]$. The inverse process happened in the universe during the first few microseconds after the big bang: the quarks and gluons were confined into hadrons. Simultaneously, the chiral symmetry of QCD was spontaneously broken. This process is intimately related to the origin of hadron masses as it corresponds to a phase transition from almost massless quarks to massive hadrons. In contrast, at large net-baryon densities and moderate temperatures, the phase transition between hadronic and partonic matter is predicted to be first order, featuring a region of phase coexistence and a critical endpoint (see the contribution of M. Stephanov in this volume). The corresponding partonic phase which contains mainly quarks and gluons (no antiquarks) is expected to prevail in the core of neutron stars [4].

Experimentally, strongly interacting matter under extreme conditions is produced and studied in high-energy heavy-ion collision experiments. At the Relativistic Heavy-Ion Collider (RHIC) at BNL experimental evidence was found for partonic degrees of freedom prevailing in the early phase of the fireball evolution. These studies will be continued at even higher energies at the Large Hadron Collider (LHC) at CERN. The goal of the experiments at RHIC and LHC is to investigate the properties of deconfined QCD matter at very high temperatures and almost zero net baryon densities. The region of the QCD phase diagram with very high net-baryon densities can be reached in heavy-ion collisions at moderate collisions energies which are and will be available at the Super-Proton-Synchrotron (SPS) at CERN, and at the Facility for Antiproton and Ion Research (FAIR) in Darmstadt. The goal of these experiments is to search for the first order phase transition and the critical endpoint. The experimental discovery of these prominent landmarks of the QCD phase diagram would be a major breakthrough in our understanding of the properties of nuclear matter. Equally important is quantitative experimental information on the properties of hadrons in dense matter which may shed light on chiral symmetry restoration and the origin of hadron masses.

At low SPS and FAIR energies also the net-baryon densities at freeze-out are the highest. This is demonstrated in figure 1 which depicts the chemical freeze-out line as function of temperature and net-baryon density as derived from an analysis of particle yields in heavy-ion collisions [7]. The numbers refer to either the total collision energy (from $2+2$ to $100+100 \mathrm{~A} \mathrm{GeV}$ ), or to laboratory kinetic energies for fixed target experiments (from 5-40 A GeV). The calculations indicate, that beam energies between 30 and $40 \mathrm{~A} \mathrm{GeV}$ (on fixed target), or total energies between $\sqrt{s}=6$ and $10 \mathrm{~A} \mathrm{GeV}$ are best suited to create the highest net-baryon densities in the laboratory. In fact, at a kinetic beam energy of $30 \mathrm{~A} \mathrm{GeV}$, structures in the excitation functions of strange-to-nonstrange particles and of the inverse slope parameters of strange particles have been observed, stimulating lively discussions on the possible onset of deconfinement.

In order to search for the first order phase transition and the QCD critical endpoint, several experimental programs are planned all over the world. The STAR and PHENIX collaborations at RHIC propose to scan the beam energies, and to search for critical phenomena [9]. For the 


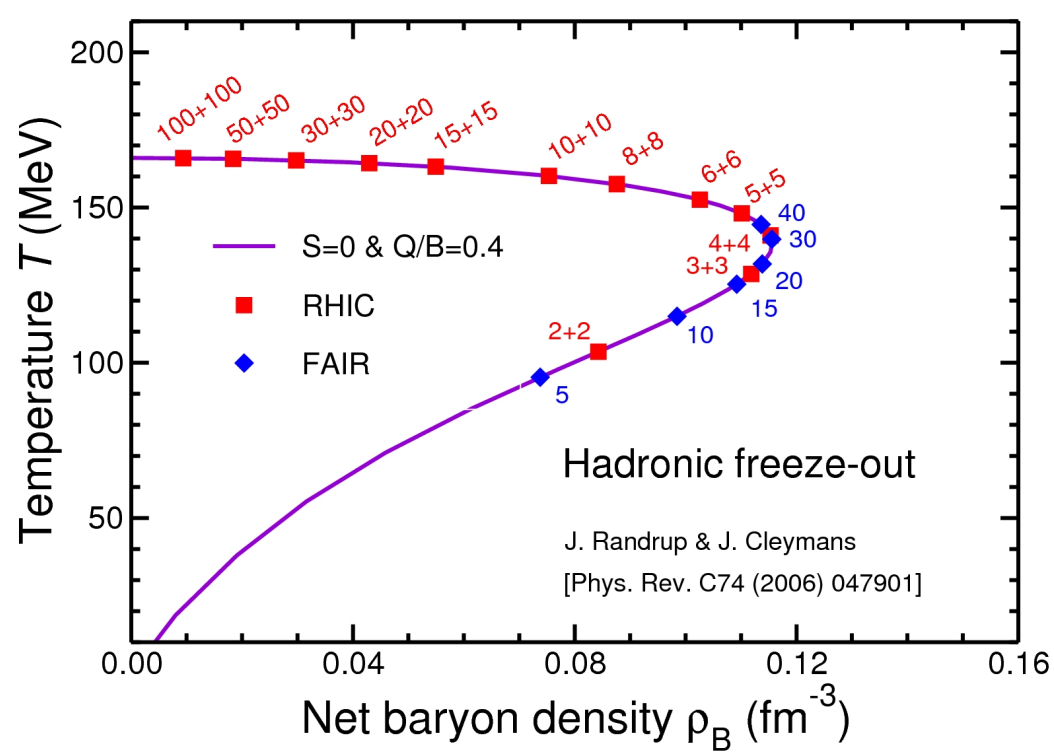

Figure 1: The hadronic freeze-out line in the plane temperature versus net-baryon density as obtained in the statistical model with the values of $\mu_{B}$ and $\mathrm{T}$ that have been extracted from the experimental data in Ref. [8]. The curve corresponds to Au+Au collisions. The symbols represent beam energies (in AGeV) at either RHIC (total energy in each beam), or FAIR (kinetic energy of the beam for a stationary target). The figure is taken from [7].

same reason, future measurements are envisaged at CERN-SPS with the upgraded NA49 detector (NA61-SHINE) using light and medium size beams [10]. At the Joint Institute for Nuclear Research (JINR) in Dubna, a heavy-ion collider project (NICA) is discussed with the goal to search for the coexistence phase of nuclear matter [11]. Due to luminosity limitations these experiments are constrained to the investigation of bulk observables which are - except for elliptic flow - predominantly sensitive to the late and dilute phase of the collision when most of the particles freeze out. In contrast, the research program of the Compressed Baryonic Matter (CBM) experiment at FAIR is focused on the measurement of diagnostic probes of the early and dense phase of the fireball evolution.

\section{Diagnostic probes of the high density fireball}

Figure 2 depicts three snapshots of the evolution of a heavy-ion collision at FAIR energies, and illustrates the time of production and eventual emission of various particle species. Particles containing charm quarks are expected to be created in the very first stage of the reaction. Then, $D$ mesons and $J / \psi$ mesons may serve as probes for the dense fireball and its degrees of freedom. Vector mesons like $\omega, \rho$ and $\phi$ mesons are produced continuously via $\pi \pi$ annihilation during the course of the reaction, and decay either again into mesons, or into a pair of leptons. However, as leptons are not affected by final-state interactions, the dileptonic decay offers the possibility to look into the fireball. In particular, the short-lived $\rho$ meson is a promising diagnostic probe of hot and dense nuclear matter. Due to their small hadronic cross sections, also multi-strange hyperons and $\phi$ mesons carry information on the dense phase of the collision, in particular via their collective 
flow. Finally, the bulk of the particles freezes out at densities below saturation density. Up to date, essentially these freeze-out probes have been measured in heavy-ion collisions at beam energies between 2 and $40 \mathrm{AGeV}$ (on stationary target).
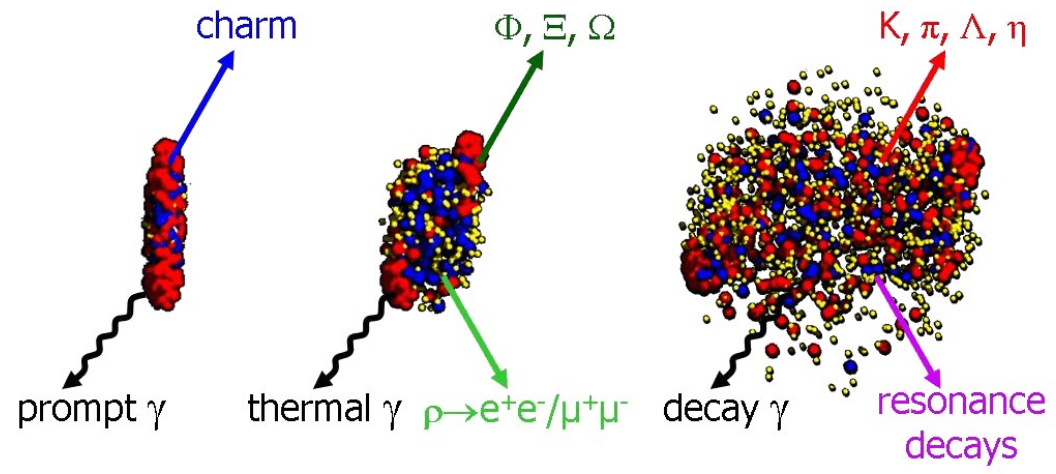

Figure 2: Sketch of the expansion phase of a $\mathrm{U}+\mathrm{U}$ collision at a laboratory beam energy of $23 \mathrm{AGeV}$ as calculated with the UrQMD model at different time steps: initial stage where the two Lorentz-contracted nuclei overlap (left), high density phase (middle), and final stage ("freeze-out") when all hadrons have been formed (right). Different particles are created in different stages of the collisions or escape from the interaction region at different times (see text). Almost 1000 charged particles are created in such a collision, most of them are pions.

The CBM experiment is designed to identify and to characterize most of the particles emitted throughout the collision. In particular particles with open and hidden charm are expected to provide valuable information about the conditions inside the dense fireball. For example, the excitation function of the charm particle ratios such as the $\psi^{\prime} /(J / \psi)$ ratio and the $(J / \psi) / D$ ratio may vary when passing the deconfinement phase transition. In addition, the initial pressure of the partonic phase influences the elliptic flow of charmonium. The transport properties of open charm mesons in dense matter - which depend on the interaction with the medium and, hence, on structure of the medium - can be studied via the yield, the elliptic flow and the momentum distributions of charmed particles. In a baryon-dominated medium these observables are expected to differ for $D$ and $\bar{D}$ mesons.

A precise measurement of the dilepton invariant mass spectrum up to about $1 \mathrm{GeV}$ provides information on the in-medium properties of the vector meson spectral function as a signal of the chiral symmetry restoration in the hot and dense matter. At higher invariant masses the spectrum contains a substantial contribution from thermal dileptons from the early partonic phase. The different origin of the dileptons is also reflected in the inverse slope of their transverse momentum spectra. The experimental determination of dileptons emitted from the high-density phase of the collision requires the measurement (and subtraction) of contributions from very early nucleonnucleon collision, from the dilute corona, and from freeze-out. The excitation function (from 2 - $45 \mathrm{AGeV}$ ) of hadron yields and phase space distributions (including multi- strange hyperons) will provide information about the fireball dynamics and the nuclear matter equation of state over a wide range of baryon densities. A non-monotonic behavior of the inverse slope as function of beam energy would signal a change in the nuclear matter properties at a certain baryon density. The inverse-slope distribution as a function of particle mass is related to the particle freeze-out time, 


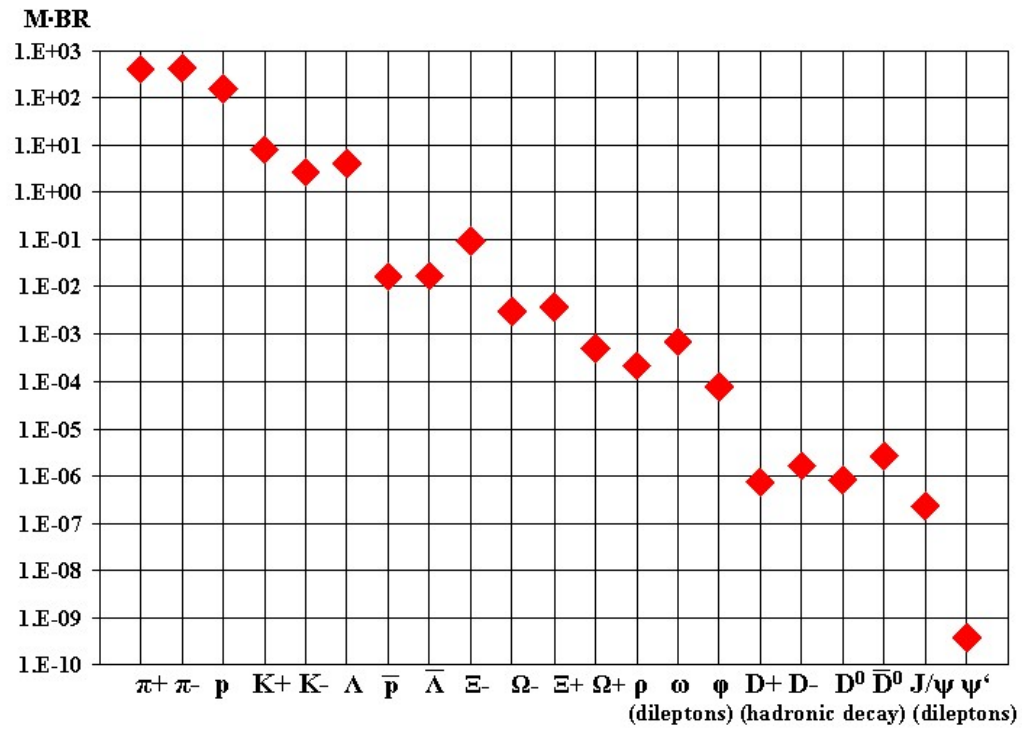

Figure 3: Particle multiplicities times branching ratio for central $\mathrm{Au}+\mathrm{Au}$ collisions at $25 \mathrm{~A} \mathrm{GeV}$ as calculated with the HSD transport code [5] and the statistical model [6]. For the vector mesons $(\rho, \omega, \phi, J / \psi$, $\psi^{\prime}$ ) the decay into lepton pairs was assumed, for D mesons the hadronic decay into kaons and pions.

and, hence, may help to disentangle the early from the late collision stages.

The strength of the elliptic flow $v_{2}$ measured as a function of transverse momentum for various particle species reflects the initial pressure of the system. The scaling of $v_{2}$ with the quark content of the particles may serve as indication for flow generation in a partonic phase. The onset (or the disappearance) of the scaling behavior at a certain beam energy would signal a change in the degrees-of-freedom of the matter. The vanishing of directed flow at a certain beam energy would indicate a strong softening of the equation-of-state.

Lattice calculations indicate large variations of the baryon, charge and strangeness susceptibilities in the vicinity of the QCD critical endpoint. These phenomena can be related to event-by-event fluctuations of conserved quantities such as net baryon number, net charge, and net strangeness. Fluctuations of the kaon, pion and proton multiplicities, in particular of their higher moments, measured as a function of beam energy, are expected to be sensitive indicators for the location of the critical point. A promising experimental task is the search for fluctuations and correlations caused by a first-order phase transition, focussing on the fact that bulk matter is unstable inside the associated spinodal region.

In this article we will focus on the capabilities of the CBM experiment with respect to rare probes such as charm and lepton pairs. In figure 3 the notation "rare probes" is quantified in terms of the product of multiplicity times branching ratio. The points are calculated for central $\mathrm{Au}+\mathrm{Au}$ collisions at $25 \mathrm{~A} \mathrm{GeV}$ using either the HSD transport code [5] or the thermal model based on the corresponding temperature and baryon-chemical potential [6]. Mesons containing charm quarks are about 9 orders of magnitude less abundant than pions (except for the $\psi$ ' meson which is even more suppressed). The dilepton decay of vector mesons is suppressed by the square of the electromagnetic coupling constant $\left.(1 / 137)^{2}\right)$, resulting in a dilepton yield which is 6 orders of 
magnitude below the pion yield, similar to the multiplicity of multi-strange anti-hyperons.

\section{The CBM experiment}

The CBM research program comprises a comprehensive scan of observables, beam energies and collision systems. The observables include low mass dilepton pairs, charmonia and open charm, but also collective flow of rare and bulk particles, correlations and fluctuations. The experimental goal is to measure these rare probes with unprecedented precision in spite of the very low multiplicities. In order to compensate for the low yields the measurements will be performed at exceptionally high reaction rates (up to $10 \mathrm{MHz}$ for certain observables). These conditions require the development of ultra fast and extreme radiation hard detectors and electronics. A particular challenge for the detectors, the front-end electronics and the data acquisition is the online selection of displaced vertices with extraordinary high speed and precision which is needed for open charm measurements.

A schematic view of the proposed CBM experimental facility is shown in figure 4. Inside a large aperture dipole magnet there is a Silicon Tracking and Vertexing System which consists of two parts: a Micro-Vertex Detector (MVD, 2 silicon pixel layers) and the Silicon Tracking System (STS, up to eight layers of silicon micro-strip detectors). The Silicon detector array has to provide the capabilities for track reconstruction, determination of primary and secondary vertices, and momentum determination. Particle identification will be performed by time-of-flight measured with a large area Resistive Plate Chamber (RPC) wall. The left panel of figure 4 depicts the setup with the Ring Imaging Cherenkov (RICH) detector for the identification of electrons from low-mass vectormeson decays. The Transition Radiation Detector (TRD) will provide charged particle tracking and the identification of high energy electrons and positrons. The Electromagnetic Calorimeter (ECAL) will be used for the identification of electrons and photons. The muon detection/hadron absorber system is shown in the right panel of figure 4. It consists of 5 double or triple stations of highly granulated gaseous micro-pattern chambers (for example Gas-Electron Multiplier (GEM) detectors) sandwiched by iron plates with a total thickness equivalent to 13 absorption lengths. The status of detector R\&D and recent results of detailed simulations are documented in [12].

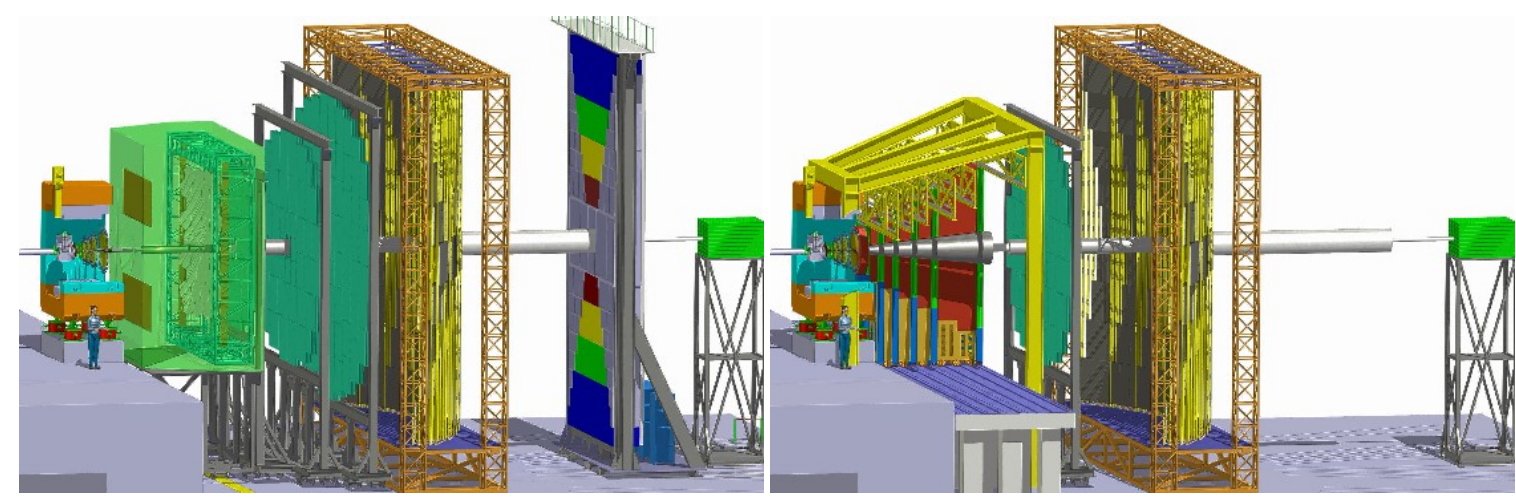

Figure 4: The CBM experimental facility with electron detectors RICH and TRD (left panel) and with the muon detection system (right panel). 
The experimental studies of dense baryonic matter at FAIR will be synchronized with the availability of the accelerators. In the first stage of the realization of FAIR the beams will be delivered by the SIS100 accelerator with energies up to $11 \mathrm{AGeV}$ for $\mathrm{Au}, 14 \mathrm{AGeV}$ for $\mathrm{Ca}$, and 29 $\mathrm{GeV}$ for protons. In order to measure electron-positron pairs in heavy-ion collisions at energies up to $8 \mathrm{AGeV}$ the HADES detector will be installed. For the measurement of multi-strange hyperons in heavy-ion collisions, and of charmed particles in proton induced reactions at SIS100, a reduced version of the CBM detector will be sufficient. Once the beams from SIS300 will be available (35 $\mathrm{AGeV}$ for $\mathrm{Au}$ and $89 \mathrm{GeV}$ for protons) the full $\mathrm{CBM}$ detector system will be ready.

\section{Feasibility studies}

The CBM detector is designed as a multi-purpose device which will be able to measure hadrons, electrons and muons in heavy-ion collisions. The optimization of the detector design is carried out through extensive feasibility studies which are performed within a newly developed software framework. The results of the simulations demonstrate that the anticipated observables including the rare probes can be measured with the proposed setup. In the following, we will present the results of the feasibility studies of charm and dilepton measurements.

\subsection{Open charm measurements}

Charm production and propagation is expected to be sensitive to the conditions in the early stage of the collision. The experimental challenge is to identify the very rare D-mesons or even $\Lambda_{c^{-}}$ baryons via their hadronic decay modes $\mathrm{D}^{0} \rightarrow K^{-} \pi^{+}$and $\mathrm{D}^{0} \rightarrow K^{-} \pi^{+} \pi^{+} \pi^{-}$, and $\mathrm{D}^{ \pm} \rightarrow K^{\mp} \pi^{ \pm} \pi^{ \pm}$, The $\mathrm{D}^{0}$ and the $\mathrm{D}^{ \pm}$have lifetimes of $124.4 \mu \mathrm{m}$ and $317 \mu \mathrm{m}$, respectively. In order to suppress the background of kaons and pions emitted from the primary vertex the high resolution Micro-Vertex Detector (MVD) is required to precisely determine the secondary decay vertex of particles with open charm. The MVD consists of two Monolithic Active Pixel Sensor(MAPS) stations located at $5 \mathrm{~cm}$ and $10 \mathrm{~cm}$ downstream of the target, with a material budget equivalent to $0.3 \% X_{0}$ and $0.5 \%$ $X_{0}$, respectively. The STS comprises 8 stations of silicon micro-strip detectors with a material budget equivalent to to $0.4 \% X_{0}$ each.

In order to reconstruct the charmed hadrons with a good signal-to-background ratio, a set of topological cuts is applied to single tracks as well as to the reconstructed vertices. Single tracks are extrapolated to the target, and are rejected if they point to the primary vertex. In contrast, the reconstructed track of the $\mathrm{D}$ meson is required to originate from the primary vertex. No kaon or pion identification with TOF is applied, however, it is important to use the time-of-flight measurement in order to reject proton tracks from the sample. The fully reconstructed invariant mass spectra of neutral and charged charmed mesons are shown in figure 5 for central $\mathrm{Au}+\mathrm{Au}$ collisions at $25 \mathrm{~A}$ $\mathrm{GeV}$ beam energy.

\subsection{Dilepton measurements}

In the following we present results of simulations which demonstrate that for FAIR energies the performance of di-electron and di-muon measurements is rather similar despite of the completely different background conditions. Electron measurements provide access to lowest invariant 

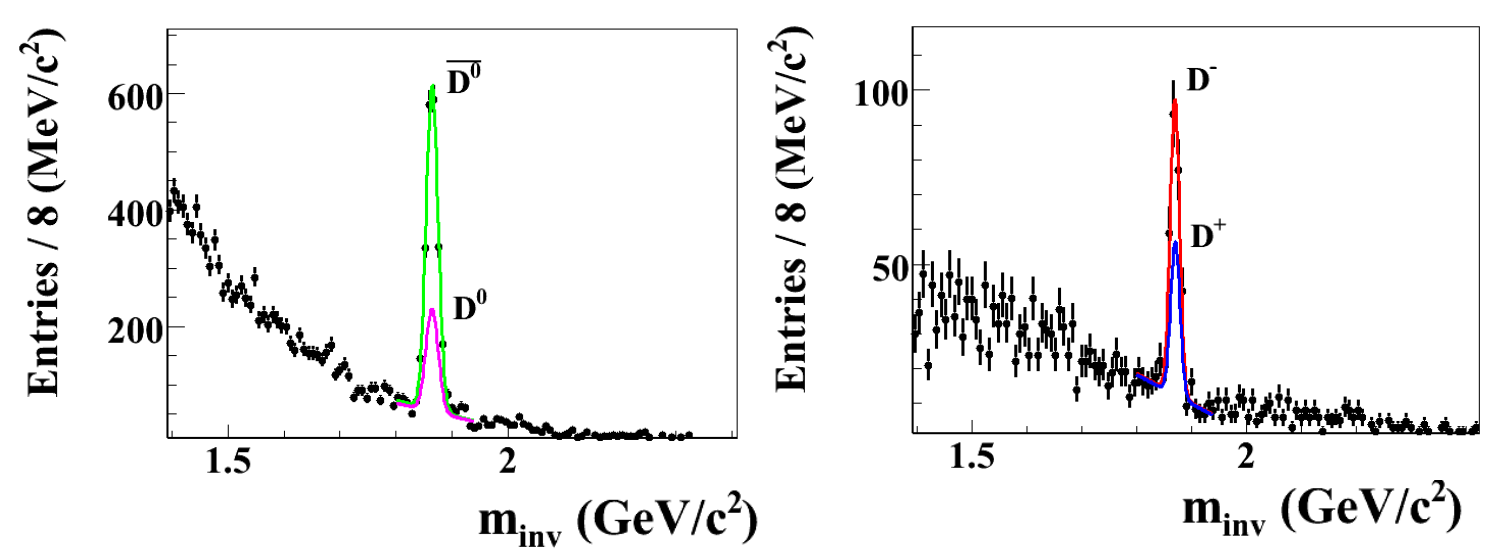

Figure 5: Invariant mass distributions of kaons and pions from D-meson decays simulated for central $\mathrm{Au}+\mathrm{Au}$ collisions at $25 \mathrm{~A} \mathrm{GeV}$. The tracking system comprises 8 stations double-sided Silicon micro-strip sensors $\left(8 \times 0.4 \% X_{0}\right)$, and 2 stations MAPS pixel sensors $\left(0.3 \% X_{0}, 0.5 \% X_{0}\right)$ at $\mathrm{z}=5 \mathrm{~cm}$ and $10 \mathrm{~cm}$. No $\mathrm{K}$ and $\pi$ identification was performed, protons were rejected via TOF. In 10 weeks about 20000 neutral $D$ mesons and 60000 charged $D$ mesons are recorded. Left panel: $D^{0}(\bar{D}) \rightarrow K \pi, c \tau=123 \mu \mathrm{m}$, Right panel: $D^{ \pm} \rightarrow K \pi \pi$, $c \tau=317 \mu \mathrm{m}$.

masses (i.e. below the mass of a muon pair), whereas muon measurements allow to generate a trigger even on low-mass vector mesons, and, hence, to collect high-statistics data. The CBM strategy is to measure both electrons and muons in order to combine the advantages of both probes, and to provide a comprehensive and consistent set of data.

In the CBM experiment electrons and positrons are identified via their Cherenkov radiation measured with the RICH, and via their transition radiation measured with the TRD stations. The Cherenkov ring positions and radii are determined by dedicated ring recognition algorithms, and the ring centers are attached to the reconstructed particle tracks. The ring radius resolution is better than 3\%. The contamination of the electron sample by misidentified hadrons is strongly reduced when the energy loss information from the TRD, and the timing information from the TOF detector is included into the analysis. In the current simulation 12 TRD layers are implemented, and the electron-to-pion separation is performed by a statistical analysis of the energy losses in each layer. The time-of-flight condition suppresses pions with momenta mainly below $1 \mathrm{GeV} / \mathrm{c}$, and rejects the kaons and protons from the electron sample. The total electron identification efficiency is $50 \%$ for $p=1 \mathrm{GeV} / \mathrm{c}$, rising to about $80 \%$ for momenta between 3 and $8 \mathrm{GeV} / \mathrm{c}$. When combining the information from RICH, TRD, and TOF the hadron yield is reduced by a factor of 10000 . If the pions are suppressed by a factor of 5000-10000, the remaining background is dominated by electrons from $\gamma$-conversion in the target and $\pi^{0}$-Dalitz decays.

The invariant mass distributions of electron-positron pairs simulated for central $\mathrm{Au}+\mathrm{Au}$ collisions at $25 \mathrm{AGeV}$ are shown in figure 6. The background is generated by UrQMD, the multiplicity of vector mesons is calculated with the HSD code. In order to reduce the electron background from $\gamma$-conversion in the target, an Au-target of $25 \mu \mathrm{m}$ is used both for low-mass vector meson and for charmonium simulations. When implementing this thin target the remaining background is dominated by electrons from $\pi^{0}$-Dalitz decays. In order to enhance the statistics for $\mathrm{J} / \psi$ mesons a 
segmented target will be used in the experiment.
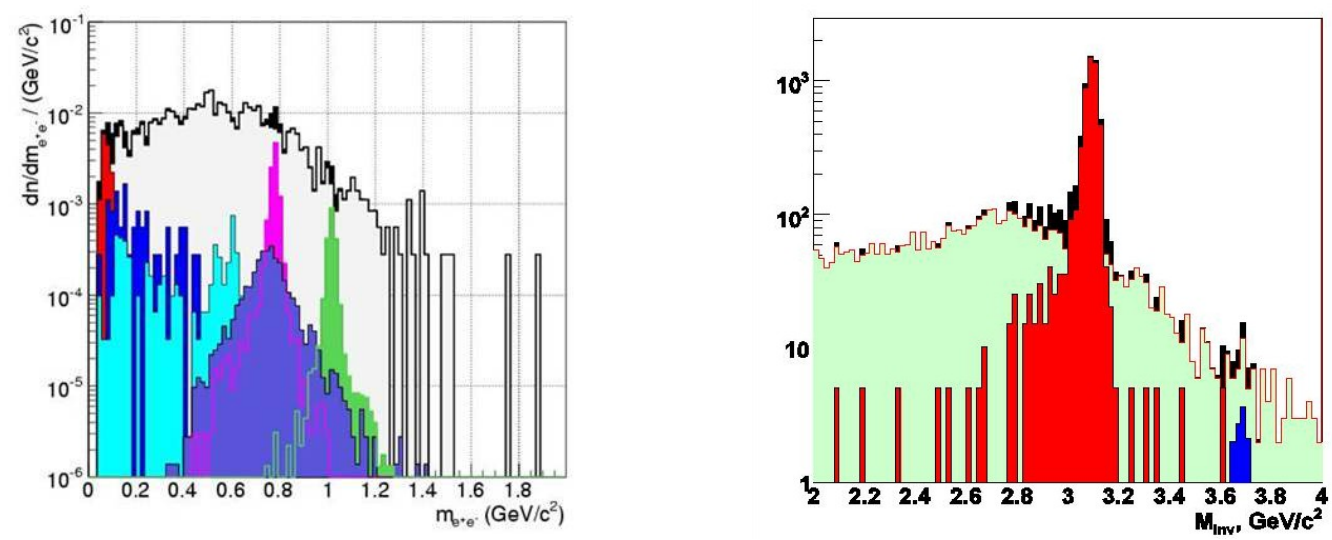

Figure 6: Invariant mass spectra of electron-positron pairs simulated for central Au+Au collisions at 25 $A \mathrm{GeV}$. The target thickness is $25 \mu \mathrm{m}$. The analysis is based on full track reconstruction (STS-TRD-TOF), and on electron identification using information from RICH (ring recognition and ring-track matching), using energy loss signals in the TRD layers, and timing information from the RPC. Left panel: low mass di-electrons simulated for 200k central collisions with a cut on $p_{t}>0.2 \mathrm{GeV} / \mathrm{c}$ for single electrons. Different sources are shown with different color; from left to right: $\pi^{0}-, \eta$-, and $\omega$-Dalitz decays, $\rho$-, $\omega$-, and $\phi$-meson. Right panel: $\mathrm{J} / \psi$ and $\psi^{\prime}$ for $4 \cdot 10^{10}$ central events, $p_{t}>1.2 \mathrm{GeV} / \mathrm{c}$ for single electrons.

The conceptual design of the muon detector system consists of 6 hadron absorber layers (iron plates of thickness 20,20,20,30,35, $100 \mathrm{~cm}$ ) and 18 gaseous tracking chambers located in triplets behind each iron slab. The absorber/detector system is placed downstream of the Silicon Tracking System (STS) which determines the particle momentum. The definition of a muon is flexible, depending on the mass of the vector meson to be identified, and on the beam energy: Muons from the decay of $J / \psi$ mesons have to pass all 6 absorber layers with a total iron thickness of $225 \mathrm{~cm}$ corresponding to 13.4 interaction length $\lambda_{I}$. The muons from the decay of low-mass vector mesons $(\rho, \omega, \phi)$ only have to penetrate through 5 iron absorber layers with a total thickness of $125 \mathrm{~cm}$ (corresponding to $7.5 \lambda_{I}$ ). The invariant mass distributions of dimuon pairs simulated for central $\mathrm{Au}+\mathrm{Au}$ collisions at $25 \mathrm{AGeV}$ are shown in figure 7. The background is generated by UrQMD, the multiplicity of vector mesons is calculated with the HSD code. The calculation performs the reconstruction of tracks measured in the Silicon Tracking System and in the muon chambers.

\section{Summary}

The CBM experiment at FAIR will enter a new era of nuclear matter research by measuring diagnostic probes never observed before at FAIR energies, and thus has a unique discovery potential. In particular, the measurement of charmed particles and dilepton pairs in heavy-ion collisions will shed light on the properties of the early and dense fireball, and on its degrees-of-freedom. In order to obtain a complete picture, a comprehensive set of observables will be measured in proton-proton, proton-nucleus, and nucleus-nucleus collisions over the full FAIR energy range. 

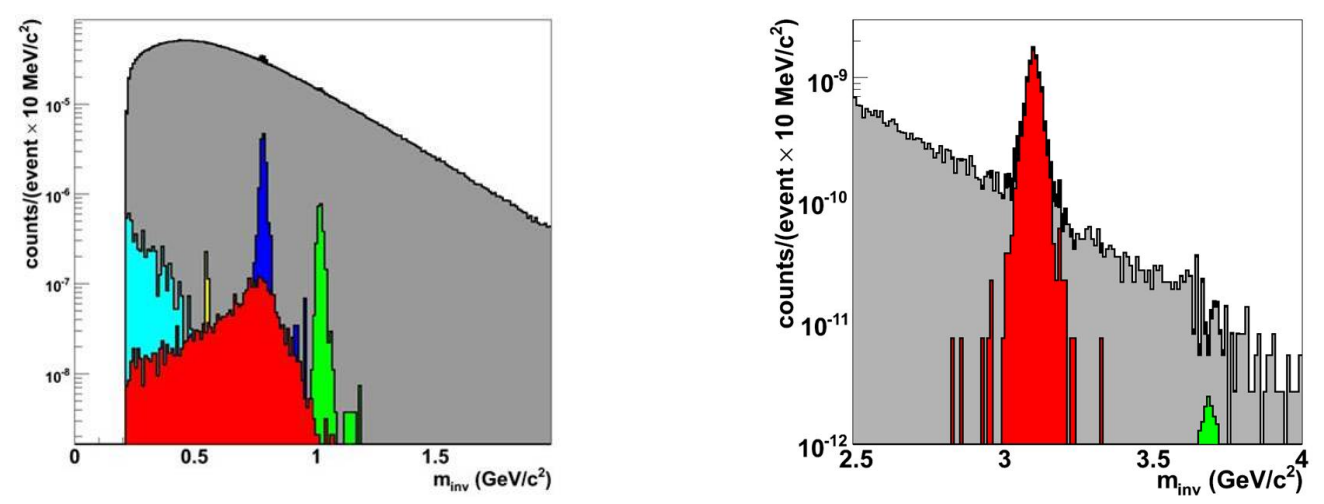

Figure 7: Invariant dimuon mass spectra calculated for $4 \cdot 10^{8}$ (left) and $3.8 \cdot 10^{10}$ (right) central $\mathrm{Au}+\mathrm{Au}$ collisions at $25 \mathrm{AGeV}$ beam energy. Left panel: low-mass range including as dimuon signals $\eta$-Dalitz decays, $\rho-, \eta-, \omega-$, and $\phi$-meson (from left to right). Right panel: charmonium mass range. A minimal transverse momentum of $p_{t}>1 \mathrm{GeV} / \mathrm{c}$ is required for single muons. No time-of-flight information is used for these spectra.

\section{Acknowledgement}

I thank my colleagues T. Galatyuk, A. Lebedev, S. Lebedev, I. Kisel, A. Kiseleva, and I. Vassiliev for performing the feasibility studies for the CBM experiment.

\section{References}

[1] F. Karsch, PoSCPOD07:026, arXiv:hep-lat/0711.0661

[2] Z. Fodor, PoSCPOD07:027, arXiv:hep-lat/0712.2930

[3] Y. Aoki, G. Endrodi, Z. Fodor, S.D. Katz, K.K. Szabo, Nature 443 (2006) 675, arXiv:hep-lat/0611014

[4] F. Weber, Prog. Part. Nucl. Phys. 54, 193 (2005), arXiv:astro-ph/0407155

[5] E. L. Bratkovskaya, private communication

[6] A. Andronic, private communication

[7] J. Randrup and J. Cleymans, Phys. Rev. C 74 (2006) 047901, arXiv:hep-ph/0607065

[8] J. Cleymans et al., Phys. Rev. C 73 (2006) 034905

[9] T. Satogata, Pos(CPOD07)051 (2007)

[10] A. Laszlo and the NA60 collaboration, Pos(CPOD07)054 (2007)

[11] V. Toneev, Pos(CPOD07)057 (2007)

[12] Eds. V. Friese and W.F.J. Müller, CBM Progress Report 2008, GSI Report 2009-03, ISBN 978-3-9811298-6-1, https://www.gsi.de/documents/DOC-2009-Feb-233.html 